\title{
Design and development of a co-planar fluorescence and X-ray tomograph
}

\author{
Juan Aguirre, Alejandro Sisniega, Jorge Ripoll, Manuel Desco, Juan José Vaquero, Senior Member, IEEE
}

\begin{abstract}
Non-contact Fluorescence Diffuse Optical Tomography (FDOT) is rapidly becoming an important tool for small animal research, since it is able to display the $3 D$ distribution of near infrared fluorescent probes in the whole body. FDOT, like other functional and molecular imaging techniques (PET, SPECT), lacks anatomical information, limiting the resolution of analyses of living systems. Furthermore, anatomical information from the subject under study can be used as a priori information for the FDOT reconstruction algorithm to improve its performance. With these goals in mind, we have built a proof-of-concept FDOT-CT hybrid machine that combines the high-resolution anatomy of $\mathrm{CT}$ with the functional information provided by FDOT.
\end{abstract}

\section{INTRODUCTION}

Jon-contact Fluorescence Diffuse Optical Tomography (FDOT) [1], [2] based in CCD technology detection in the absence of matching fluids, is emerging as an important biomedical tool due to its ability to retrieve the spatial distribution of the concentration of fluorophores in small animals in vivo not invasively.

However, FDOT images lacks of morphological landmarks that may help to identify the underlying anatomical structures. In this situation a co-planar X-ray Computerized Tomography (CT) system can provide very useful anatomical information. Even more, this set-up is an optimal platform to test many improvements in FDOT performance by using the tissue distribution given by CT as a priori information for the FDOT reconstruction algorithm

\section{MATERIALS AND METHODS}

All the FDOT and CT components were attached to a rotating gantry as depicted in Fig. 1. Regarding the FDOT system, the light emerging from a diode laser is focused into the sample at predefined surface points using two mirrors moved by galvanometers. The sample is slightly compressed between two antireflective plates, allowing two angular projections when the CCD chip is positioned parallel to the plates. Typically 100 equidistant sources inside a rectangle for each angular projection are chosen.

Manuscript received November 14, 200. This work is partially funded by the CD-TEAM Project, CENIT Program, Spanish Ministerio de Industria grants from the Ministerio de Educación y Ciencia, projects TEC2007-64731 and TEC2008-06715-C02-01 and by Caja Navarra.

J. Aguirre, A. Sisniega, M. Desco and JJ. Vaquero are with the Unidad de Medicina y Cirugía Experimental Hospital General Universitario Gregorio Marañón, Madrid Spain (J.J. Vaquero e-mail: juanjo@hggm.es).

J. Ripoll is with the Institute of Electronic Structure \& Laser - FORTH Heraklion. Greece.
The excitation light and the fluorescence light at the surface of the sample are collected with a cooled, low-noise CCD camera using the appropriate filters. Typically 100 virtual detectors for each projection are selected; the normalized Born field [3] is then calculated for the reconstruction process. Accordingly to the slab-like geometry of the sample, the forward problem is solved by the source image method for planar boundaries under the diffusion approximation. All the process is controlled via software written in $\mathrm{C}++$ and IDL code. To solve the inverse problem the sample is digitalized in a cubic mesh with voxel volume of $0.5 \mathrm{~mm}^{3}$. The ART algorithm with positive restriction is employed to make the reconstructions.

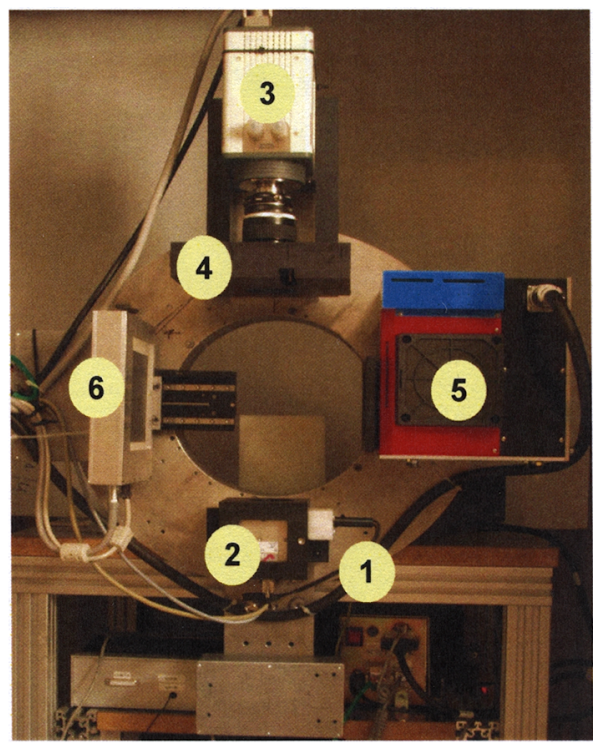

Fig. 1. FDOT-XCT experimental setup. 1)Diode laser. 2) Scan Cube. 3) CCD Camera. 4) Motorized filter wheel. 5) X-ray source. 6) X ray detector.

The CT system consists of a microfocus X-ray source and a flat-panel type digital detector diagonally opposed (cone-beam geometry), and attached to a rotating gantry, providing a FOV large enough acquire an average-sized mouse with a single bed position. A modified FDK algorithm is used to reconstruct the acquired volume. 


\section{RESULTS}

Fig. 2 shows FDOT reconstructed slices of a capillary (inner diameter $<1 \mathrm{~mm}$ ) filled with Alexa Fluor 750, inside an agar slab phantom $\left(5 \times 5 \times 1.5 \mathrm{~cm}^{3}\right)$, with $\mu \mathrm{a}=0.3 \mathrm{~cm}^{-1}$ and $\mu$ 's $=10$ $\mathrm{cm}^{-1}$ [5]. Data acquisition was done using one geometrical projection; $7 \times 7$ sources and $15 \times 15$ detectors were selected, equally spaced in a $2 \times 3 \mathrm{~cm}^{2}$ area. A cubic mesh of $20 \times 20 \times 10$ elements was chosen for the tomographic reconstruction, using 15 ART iterations, which took less than 1 minute using a standard PC.

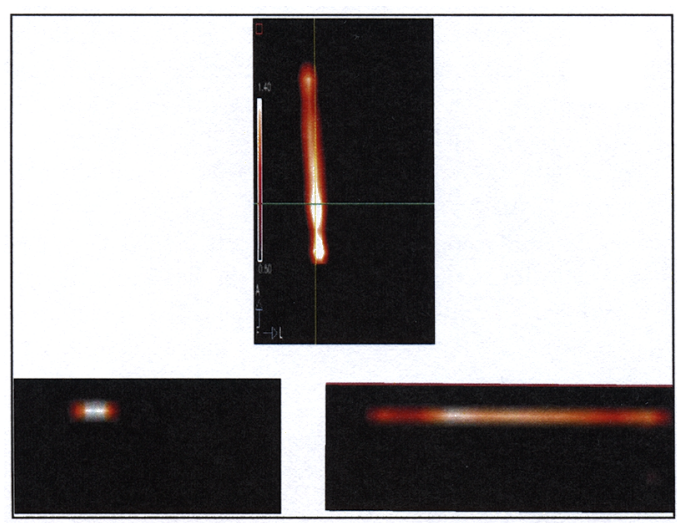

Fig 2. Up: Coronal slice of the FDOT reconstructed image, the capillary can be clearly seen. The green line corresponds to the axial slice presented at the bottom left corner and the yellow line correspond to the sagittal slice presented on the bottom right corner.

Fig. 3 shows a rendered 3D image of a fused FDOT image (in red color scale) with a XCT image. In red appears the FDOT reconstructed image of a capillary (inner diameter $<1 \mathrm{~mm}$ ) filled with Alexa Fluor 750 witch was inserted in the esophagus of a euthanized one week year old rat. In gray color scale appears the bone structure of the subject given by the CT reconstruction conveniently thresholded.

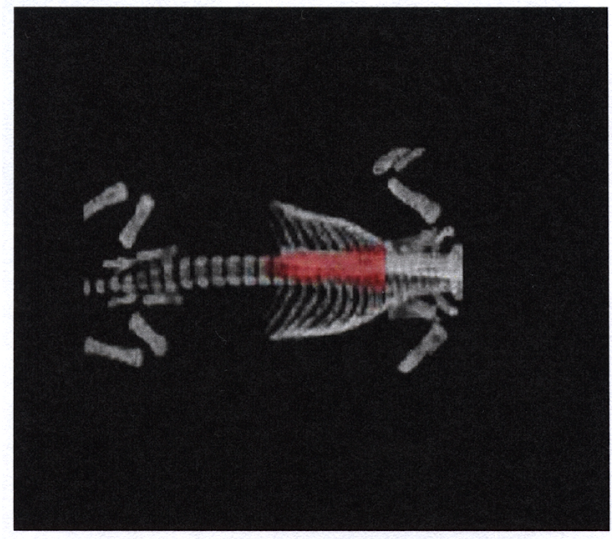

Fig. 3. FDOT-XCT of a 1 week old rat with a capillary filled with Alexa Fluor 750 inserted in the esophagus.

\section{REFERENCES}

[1] V. Ntziachristos, J.Ripoll, L. Wang, R. Weissleder, "Looking and listening to light: the evolution of whole-body photonic imaging," Nat. Biotechnol., vol. 23, no. 3, pp. 313-320, March. 2005.

[2] J. Ripoll, R. Schulz and V. Ntziachristos, "Free-space propagation of diffuse light: theory and experiments," Phys. Rev. Lett, 2003. vol. 91, no. 10, pp. 103901 . September. 2003.

[3] V. Ntziachristos and R. Weissleder, "Experimental three-dimensional fluorescence reconstruction of diffuse media by use of a normalized Born approximation," Opt. Lett, 2001. vol. 26, no. 12, pp. 893-5. June. 2001

[4] R. Cubbedu et al, "A solid tissue phantom for photon migration studies," Phys.Med. Biol, vol. 42 p. 1971-1979. November.1997. 